\title{
Acute Pancreatitis Following Brentuximab Vedotin Therapy for Refractory Hodgkin Lymphoma: A Case Report
}

\author{
S. A. M. Urru $\cdot$ E. Mariotti $\cdot$ P. Carta $\cdot$ \\ S. Massidda $\cdot$ M. Marcias $\cdot$ R. Murru $\cdot$ \\ P. Sanna $\cdot$ E. Angelucci
}

Published online: 4 February 2014

(c) The Author(s) 2014. This article is published with open access at Springerlink.com

Brentuximab vedotin is an antibody drug conjugate recently approved for the treatment of adult patients with relapsed or refractory Hodgkin lymphoma.

Here, we present a patient with brentuximab vedotinassociated pancreatitis diagnosed on the basis of clinical and radiologic findings and laboratory data. To our knowledge there have been no published reports of pancreatitis occurring with this medication.

A 65 year old white man was diagnosed in December 2011 with Hodgkin lymphoma, mixed cellularity subtype, stage IIa, non-bulky disease involving abdominal sites, without retroperitoneal lymph node involvement. The patient denied a personal or family history of gastrointestinal disease, smoking, or alcohol abuse and was not obese.

From January to July 2012, the patient received six standard cycles of adriamycin, bleomycin, vinblastine, and dacarbazine treatment and, because of lymphoma refractoriness, from November to January 2013 four cycles of ifosfamide, gemcitabine, vinorelbine, and prednisone salvage therapy, without experiencing any gastrointestinal disorder. Unfortunately, post-chemotherapy computed tomography, positron emission tomography, and inguinal lymph node

\section{S. A. M. Urru ( $\square)$}

CRS4, Biomedicine Sector, Center for Advanced Studies, Research and Development in Sardinia Technology Park Polaris, Building 1 Piscina Manna, Pula, 09010 Cagliari, Italy

e-mail: silvanaurru@crs4.it

E. Mariotti - P. Carta · M. Marcias - P. Sanna Antiblastic Drugs Unit-Pharmacy Department, Ospedale Oncologico di Riferimento Regionale "Armando Businco", Via E. Jenner 1, 09100 Cagliari, Italy

S. Massidda $\cdot$ R. Murru $\cdot$ E. Angelucci

Hematology and BMT Unit, Ospedale Oncologico

di Riferimento Regionale "Armando Businco",

Via E. Jenner 1, 09121 Cagliari, Italy biopsy showed disease progression. Therefore, on April 2013, the patient began treatment with $1.8 \mathrm{mg} / \mathrm{kg}$ brentuximab vedotin total dose $150 \mathrm{mg}$, intravenously once every 3 weeks. The patient did not receive premedication.

Laboratory tests after the first administration showed an increase in aspartate aminotransferase, alanine aminotransferase, and gamma glutamyl transferase levels that normalized within a few days.

A few days after the second brentuximab vedotin infusion, the patient developed nausea, stypsis, and epigastric pain. He was hospitalized 7 days after the second administration of brentuximab with persistent nausea, abdominal tenderness, dehydration, and acute constant pain in the epigastric area that gradually worsened. He was afebrile and his only medication was lansoprazole. Abdomen ultrasound examination was negative for gallstones.

Additional findings were severe neutropenia (absolute neutrophil count $100 / \mu \mathrm{L}$ ) and a significant increase in amylase and lipase levels of 206 and $429 \mathrm{U} / \mathrm{L}$, respectively (amylase upper limit of normal values $52 \mathrm{U} / \mathrm{L}$ and lipase upper limit of normal values $61 \mathrm{U} / \mathrm{L}$ ), (Fig. 1). Amylase elevation was consistent with grade 3 toxicity, whereas serum lipase was consistent with grade 4 (MedDRA code 10040139). Bilirubin and transaminase levels were from two to three times higher than normal levels. A diagnosis of druginduced acute pancreatitis was made supported by serum amylase levels three times above the upper limit of normal, as reported in the literature [1]. Moreover, abdomen computed tomography showed limited iliac-inguinal nodes with lymphoma involvement, excluding pancreas lymphoma infiltration as the cause of the pancreatitis. The patient was given intravenous fluids, antibiotics, and granulocyte colony-stimulating factor until resolution of neutropenia.

Pancreas enzymes returned to within normal levels in 3 weeks and the third cycle of brentuximab vedotin was 
Fig. 1 Serum amylase and lipase levels during brentuximab vedotin therapy

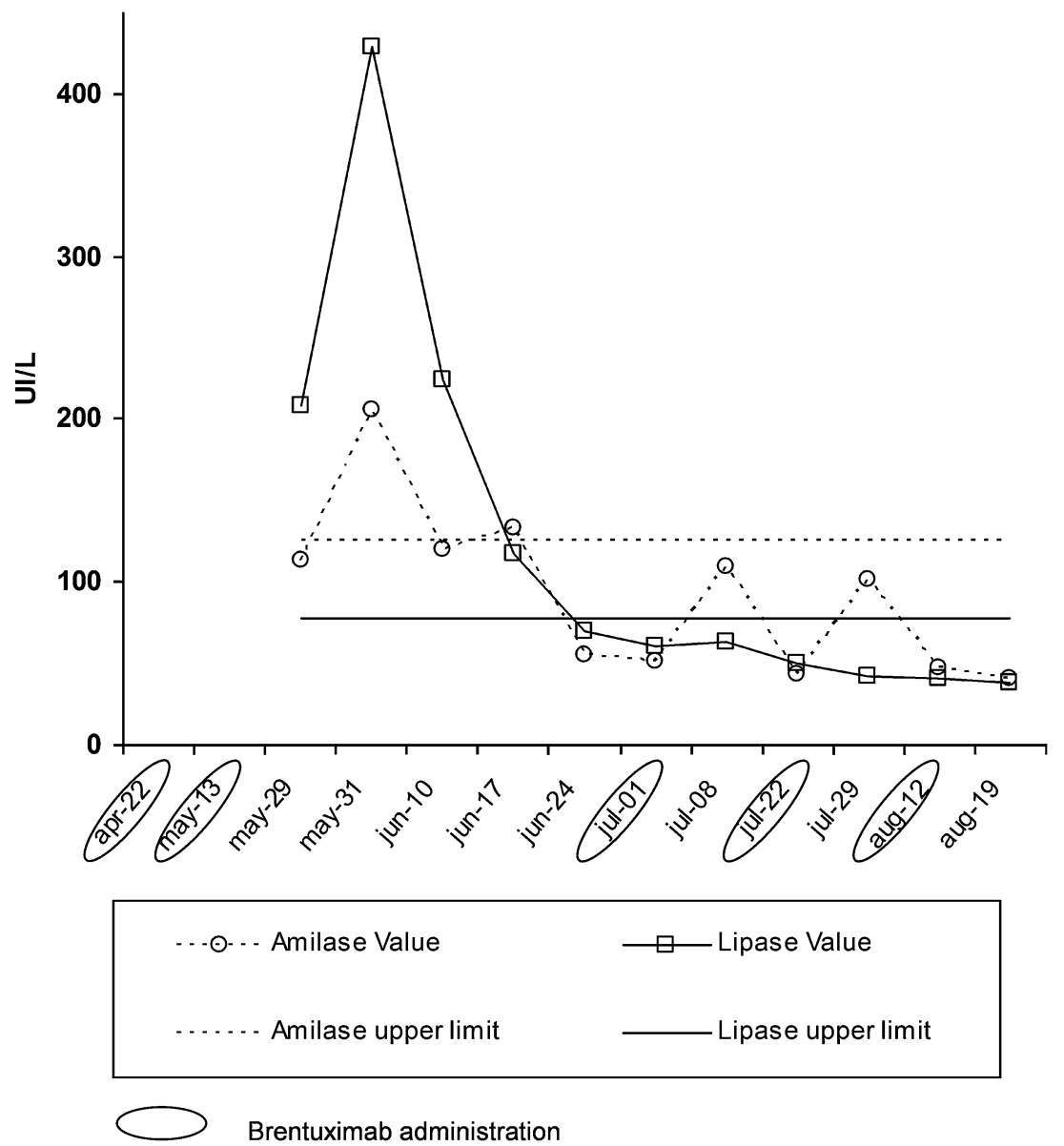

given at the same dose at 50 days from the second infusion and at 30 days from the onset of acute pancreatitis. Administration of subsequent chemotherapy cycles was decided based on improvement of clinical conditions, normalization of amylase and lipase values, and partial reduction of abdominal nodes (abdominal US). After every brentuximab vedotin administration, the patient required subcutaneous granulocyte colony-stimulating factor for 4 days to prevent neutropenia but did not present with any other severe adverse event. No recurrence of pancreatitis or any other side effect was recorded.

At the time of writing, after six cycles of treatment with brentuximab vedotin, the patient experienced disease progression. According to Naranjo's algorithm [2], the causal relationship between medication and acute pancreatitis is probable ( score $=5$ ), although this potential adverse event is rare and no other increase in amylase and lipase levels was reported after brentuximab re-challenge.

Acute pancreatitis is a reversible inflammatory process of the pancreas. Although the disease process may be limited to pancreatic tissue, it can also involve peripancreatic tissues or more distant organ sites [3]. Although drug-induced acute pancreatitis is considered a rare diagnosis with an estimated incidence of 0.1-2 \% [4], many other antineoplastic drugs have been associated with pancreatitis $[5,6]$.

An extensive review of the literature does not reveal other cases of brentuximab vedotin-induced pancreatitis.

As the number of clinical studies is increasing with this new promising drug (37 open studies, http://www. clinicaltrials.gov), we suggest amylase and lipase levels determination when a patient experiences prolonged nausea and/or vomiting and abdominal pain. Moreover, in light of the seriousness of the disease, hematologists should be alert to the possibility of such an adverse reaction.

This case has been reported to the Italian Health Authority (AIFA) registered as number 212194 on July 2013 and to the manufacturer of the drug (Takeda).

Conflict of interest We have no conflicts of interest to disclose.

Open Access This article is distributed under the terms of the Creative Commons Attribution Noncommercial License which permits any noncommercial use, distribution, and reproduction in any medium, provided the original author(s) and the source are credited. 


\section{References}

1. Cotton PB, Lehman G, Vennes J, Geenen JE, Russell RC, Meyers WC, Liguory C, Nickl N. Endoscopic sphincterotomy complications and their management: an attempt at consensus. Gastrointest Endosc. 1991;37:383-93.

2. Naranjo CA, Busto U, Sellers EM. A method for estimating the probability of adverse drug reactions. Clin Pharmacol Ther. 1981;30(2):239-45.
3. Carroll JK, Herrick B, Gipson T, Lee SP. Acute pancreatitis: diagnosis, prognosis, and treatment. Am Fam Physician. 2007;75(10):1513-20.

4. Nitsche CJ, Jamieson N, Lerch MM, Mayerle JV. Drug induced pancreatitis. Best Prac Res Clin Gastroenterol. 2010;24:143-55.

5. Underwood TW, Frye CB. Drug-induced pancreatitis. Clin Pharm. 1993;12(6):440-448.

6. Tester W, Forbes W, Leighton J. Vinorelbine-induced pancreatitis: a case report. J Natl Cancer Inst. 1997;89(21):1631. 